\title{
Correlation between placental pathology and neonatal morbidity: a case-control study
}

\author{
Anna Kornete ${ }^{1 *}$, Natalija Vedmedovska ${ }^{1,2}$, Solvita Blazuka $^{3}$
}

\author{
${ }^{1}$ Department of Obstetrics and Gynecology, Riga Stradins University Riga, Latvia \\ ${ }^{2}$ Department of Prenatal Diagnostic, Riga Maternity Hospital, Riga, Latvia \\ ${ }^{3}$ Department of Pathology, Vidzemes Hospital, Valmiera, Latvia
}

Received: 01 December 2016

Accepted: 26 December 2016

*Correspondence:

Dr. Anna Kornete,

E-mail: annakornete@gmail.com

Copyright: (C) the author(s), publisher and licensee Medip Academy. This is an open-access article distributed under the terms of the Creative Commons Attribution Non-Commercial License, which permits unrestricted non-commercial use, distribution, and reproduction in any medium, provided the original work is properly cited.

\begin{abstract}
Background: Preterm births occur in approximately $12 \%$ of pregnancies worldwide and in $5.6 \%$ of pregnancies in Latvia, and the incidence has increased. Prematurity poses the major challenge in perinatology and pediatrics, accounting for $75 \%$ of perinatal mortalities and $50 \%$ of long-term complication. The placenta is a unique organ in explaining the incomprehensible pathogenesis of prematurity.

Methods: The retrospective case-control study was conducted to determine placental histological and microbiological findings associated with gestational age and neonatal morbidity.

Results: Histological chorioamnionitis was the most prevalent lesion in extremely preterm and very preterm birth groups compared with moderate to late preterm and term birth groups $(\mathrm{P}=0.027)$. A higher rate of funisitis was detected among extremely preterm and very preterm birth cases $(\mathrm{P}=0.001)$. Microbiological examination of placentas in preterm birth cases most commonly revealed Streptococcus agalactiae, Staphylococcus epidermidis, Staphylococcus haemolyticus. Umbilical cord vessels thrombosis and placental thrombotic vasculopathy were found mostly in moderate to late preterm birth category $(\mathrm{P}=0.032 ; \mathrm{P}=0.008$, respectively). Intrauterine growth restriction was linked to chorionic villous edema $(\mathrm{P}=0.007)$ and chorionic villous fibrinoid necrosis $(\mathrm{P}=0.014)$. Chorion-decidual hemorrhage and deciduitis were significantly associated with respiratory distress syndrome $(\mathrm{P}=0.036 ; \mathrm{P}=0.022$, respectively). Chorion-decidual hemorrhage was the main predisposing factor for hypoxic-ischemic encephalopathy $(\mathrm{P}=0.058)$.

Conclusions: Comprehension of the pathogenic mechanisms of prematurity of the placenta and preterm births, and the impact of placental prematurity on neonatal morbidity may lead to improved prenatal diagnostic and enhanced preventive care for both the mother and the child.
\end{abstract}

Keywords: Histology, Neonatal morbidity, Placenta, Preterm birth

\section{INTRODUCTION}

Preterm births occur in approximately $12 \%$ of pregnancies worldwide and in $5.6 \%$ of pregnancies in Latvia, and the incidence has increased. ${ }^{1,2}$ The World Health Organization estimates there is 15 million preterm births globally and 1 million direct fatalities annually. ${ }^{1}$ Prematurity poses the major challenge in perinatology and pediatrics, accounting for $75 \%$ of perinatal mortalities and $50 \%$ of long-term complication. ${ }^{3}$ The complications of prematurity include adverse cognitive, organ functional and motor outcomes. ${ }^{4}$

Placental pathology is described in two clinical categories of preterm births: spontaneous preterm births and induced preterm births. Spontaneous preterm births can be 
classified into two separate clinical scenarios: (i) premature onset of labor defined as regular contractions with accompanying cervical change and with intact membranes, accounting for $40-45 \%$ of cases of preterm births or (ii) preterm premature rupture of membranes defined as spontaneous rupture of membranes at less than 37 weeks of gestation and at least one hour before the onset of contractions, seen in $25-30 \%$ of preterm births. ${ }^{5}$ Induced preterm birth is defined when the labor is induced or caesarean section is performed for maternal or fetal indications. ${ }^{5,6}$ Histological chorioamnionitis is the most common lesion reported in placenta in spontaneous preterm births. ${ }^{5,6}$ While in almost $30 \%$ of cases a clinical cause is not identified. 5 Nevertheless, it is clear that placental pathology has been implicated in the pathogenesis of preterm birth and neonatal morbidity. ${ }^{7}$

Clinical management to reduce the incidence of preterm births have not been yet very successful. ${ }^{8}$ Prevention and treatment for individual risk factors have largely been targeting, focusing on answering clinical questions rather than pathogenic mechanisms, including placental ones. ${ }^{8}$ Placental pathological assessment in these cases may provide valuable clues for predicting which neonate and why some neonate may be at relatively higher risk for developing short-term and long-term complications. ${ }^{8}$ Thus, placental histological examination may provide a significant impact on the care of the prematurely born neonate and insights into future investigations. ${ }^{7,8}$

The retrospective case-control study was conducted to determine placental histological and microbiological pathology associated with gestational age and neonatal morbidity.

\section{METHODS}

All singleton preterm birth cases when placental pathological assessment was done in Vidzemes hospital in Valmiera, Latvia, from January 2010 through July 2016 were included in the retrospective case-control study. Preterm birth cohort $(\mathrm{N}=72)$ was subcategorized according to gestational age at the time of birth: 17 extremely preterm (22-27 weeks' gestation), 17 very preterm (28-31 weeks' gestation) and 38 moderate to late preterm (32-36 weeks' gestation). Twenty term births (3741 weeks' gestation) were selected as control group. Inclusion criteria for controls were: primigravida, nonsmoker mother without any systemic diseases, singleton pregnancy and vaginal delivery.

Placental pathology reports from preterm birth cases were obtained and reviewed. Due to variations in classification of placental histology over the enrollment period, and to minimize interobserver variability, placental specimens were rereviewed. Slides from samples of each case placenta were rereviewed by a single pathologist, blind to clinical history prior pathologic diagnoses and gestational age at birth. Placentas were examined in accordance with the guidelines published by the Royal College of
Obstetricians and Gynaecologists and the Royal College of Pathologists, and the College of American Pathologists. ${ }^{9,10}$ Five micron sections of embedded tissues were deparaffinized with xylene and ethanol and stained with hematoxylin and eosin and evaluated in a magnification of 100-200 times for different histopathologic findings of membranes, placental disc and umbilical cord tissues., ${ }^{9,10}$ Afterwards placental histological findings were linked to data in the maternal and fetal medical documentation.

Histological chorioamnionitis was defined as amniotropic infiltration by both maternal and fetal neutrophils in the chorioamniotic membranes due to microbial invasion of the amniotic cavity. ${ }^{11}$ The diagnosis of deciduitis was given when lymphoplasmacytic infiltrate was present in the decidua of the basal plate. ${ }^{11}$ In case inflammatory process involved the umbilical cord (umbilical vein, umbilical artery and the Wharton's jelly), this was referred as funisitis. ${ }^{12}$ In chorionic villous edema microscopically the villous stroma was edematous and chorionic villi appeared enlarged, syncytiotrophoblast cell necrosis, reduced chorionic villous capillary lumens, and increased number of Hofbauer cell, increased circulating nucleated fetal red blood cells were present. ${ }^{13}$ The histopathologic features of degenerative changes in the chorionic villous (villous fibrosis, hyalinization, calcification and necrosis) tree due to sustained proximal vascular occlusion included circulatory stasis with villous stromal-vascular karyorrhexis which was degeneration of red blood cells, endothelial cells, and villous stromal fibroblasts, avascular chorionic villi with depositions. ${ }^{5}$ Placental thrombotic vasculopathy was defined by the presence of thrombosis in chorionic plate and chorionic villous vessels led to degeneration and eventual loss of capillaries in more than 15 affected chorionic villi per section. ${ }^{5}$ In umbilical cord vessels thrombosis formation or presence of a blood clot was found in the umbilical vein or either or both the umbilical arteries. ${ }^{5}$

The neonatal morbidities as intrauterine growth restriction (IUGR), intraventricular hemorrhage (IVH), respiratory distress syndrome (RDS), hypoxic-ischemic encephalopathy (HIE) and sepsis were analyzed within the study. IUGR was identified by serial ultrasonography scans including biometric and Doppler measurements. ${ }^{6}$ IVH was diagnosed by cranial ultrasonography shortly after delivery. ${ }^{6}$ The diagnosis of RDS, HIE and sepsis was confirmed by clinical and laboratory assessments. ${ }^{6}$ All the grades of IUGR, IVH, RDS, HIE and sepsis were deemed as either absent or present since the lack of data for individual analyze of the results for each grade.

The Chi-square test was used to determine the difference in proportion of neonatal morbidities and placental lesions in each gestational age categories. Multiple regression analysis was used to define the likelihood of single placental lesion being contributory to the development of prematurely born neonatal morbidity. Statistical data were analyzed with statistical data 
programme IBM SPSS Statistics. P-values <0.05 was considered statistically significant.

\section{RESULTS}

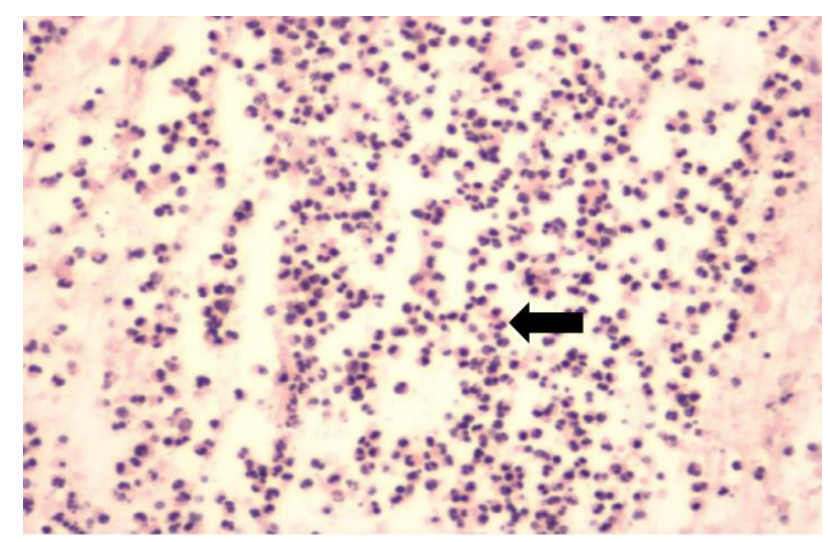

Figure 1: Chorionamnionitis. Diffuse intense neutrophilic infiltration of amnion and chorion. Hematoxillin and eosin stain; $200 \mathrm{x}$.

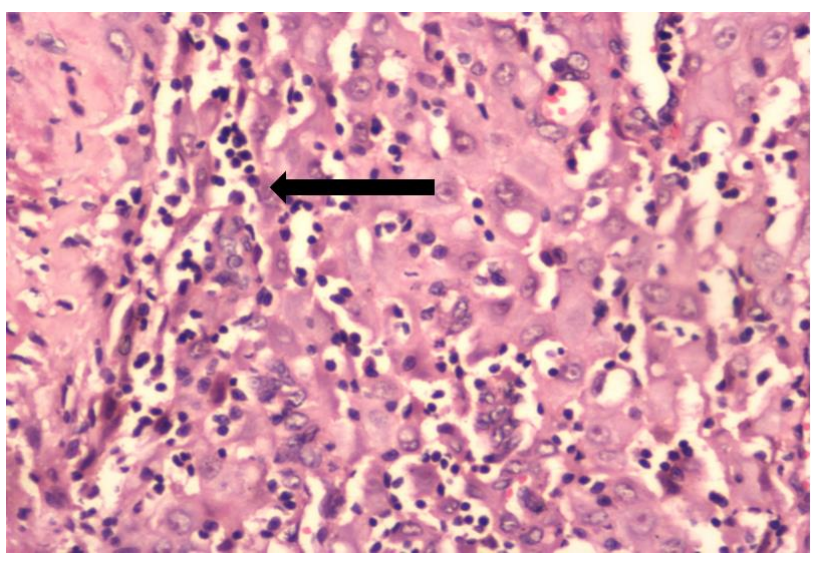

Figure 2: Deciduitis. Moderate leukocytic infiltration of decidual tissue. Hematoxillin and eosin stain; $100 \mathrm{x}$.

Histological chorioamnionitis (Figure 1) was the most prevalent lesion in extremely preterm and very preterm birth groups compared with moderate to late preterm and term birth groups $(41.2 \%$ vs. $47.1 \%$ vs. $26.3 \%$ vs. $7.2 \%$, respectively; $\mathrm{P}=0.027)$. Clinical chorioamnionitis was diagnosed in four $(15.3 \%)$ cases out of the total 26 histological verified chorioamnionitis; in nine $(34.6 \%)$ cases severe chorioamnionitis was detected characterized with formation of microabscesses under the chorion. Deciduitis (Figure 2) was found more often in extremely preterm and very preterm birth categories than in moderate to late preterm and term birth categories $(29.4 \%$ vs. $17.6 \%$ vs. $7.9 \%$ vs. $4.8 \%$, respectively; $\mathrm{P}=0.014$ ). A higher rate of funisitis (Figure 3) was detected among extremely preterm and very preterm birth cases compared with moderate to late preterm and term birth cases (17.6\% vs. $13.2 \%$ vs $7.9 \%$ vs. $5.0 \%$, respectively; $\mathrm{P}=0.001)$.

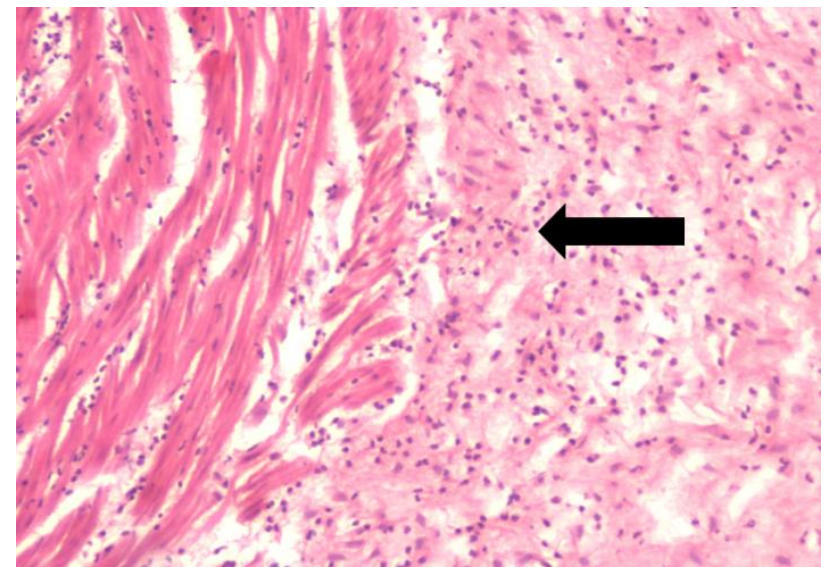

Figure 3: Funisitis. Diffuse neutrophilic infiltration around umbilical vessels. Hematoxillin and eosin stain; $200 \mathrm{x}$.

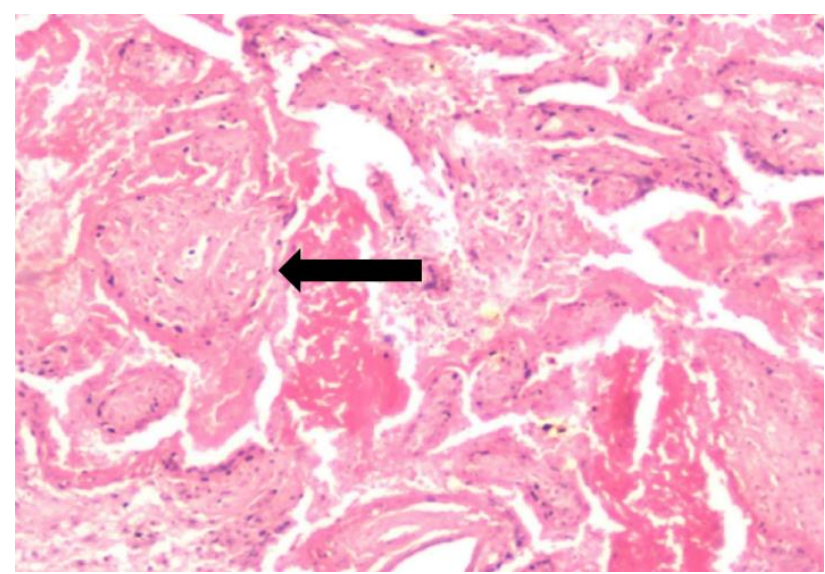

Figure 4: Placental thrombotic vasculopathy.

Chorionic villi with hyalinization and calcification in stroma. Hematoxillin and eosin stain; $200 \mathrm{x}$.

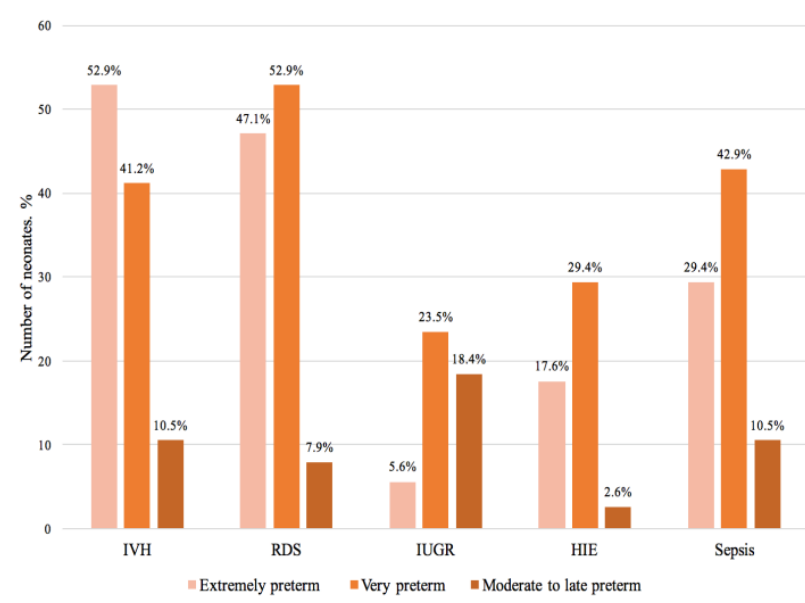

Figure 5: Neonatal morbidity (intraventricular hemorrhage, IVH; respiratory distress syndrome, RDS, intrauterine growth restriction, IUGR; hypoxicischemic encephalopathy, HIE and sepsis) in the preterm birth categories. 
Microbiological examination of placenta in cases of preterm birth revealed that the most common pathogens were: Streptococcus agalactiae (12.7 \%), Staphylococcus epidermidis $(9.7 \%)$ and Staphylococcus haemolyticus (8.3\%). Streptococcus viridans (6.9\%), Escherichia coli (6.9\%), Enterobacter cloacae (5.6\%), Staphylococcus hominis (4.2\%), Staphylococcus simulans (4.2\%) and Gardnerella vaginalis $(2.7 \%)$ were noticed as well. In controls no bacteriological culture was isolated in $80.0 \%$ of placentas, while $20.0 \%$ Staphylococcus epidermidis, Staphylococcus hominis, Streptococcus viridans and Escherichia coli were found.

Chorionic villous edema was diagnosed predominantly in many cases with the same frequency in both extremely preterm and very preterm birth groups $(41.2 \%$ vs. $41.2 \%$, respectively); it was statistically more significant comparing with moderate to late preterm and term birth categories $(26.4 \%$ vs. $5.0 \%$, respectively; $\mathrm{P}=0.023)$. The majority of placentas with chorionic villous edema concomitantly demonstrated chorioamnionitis $(78.4 \%)$.

Umbilical cord vessels thrombosis was found mostly in moderate to late preterm birth category compared with extremely preterm and very preterm and term birth categories $(5.9 \%$ vs. $5.9 \%$ vs. $21.1 \%$ vs. $10.0 \%$, respectively; $\mathrm{P}=0.032)$ Placental thrombotic vasculopathy (Figure 4) was more prevalent in moderate to late preterm birth cases than in extremely preterm and very preterm, and term birth cases ( $11.8 \%$ vs. $23.5 \%$ vs. $31.6 \%$ vs. $10.0 \%$, respectively; $\mathrm{P}=0.008)$. Chorionic villous fibrosis prevailed in moderate to late preterm and term birth groups $(68.4 \%$ vs. $65.0 \%$, respectively) when compared with extremely preterm and very preterm birth groups $(41.2 \%$ vs. $41.2 \%$, respectively; $\mathrm{P}=0.014)$. A higher rate of chorionic villous hyalinization was detected among moderate to late preterm and term birth groups compared with extremely preterm and very preterm birth groups $(57.9 \%$ and $55.0 \%$ vs. $29.4 \%$ vs. $17.6 \%$, respectively; $\mathrm{P}=0.055)$. The same association was noticed between chorionic villous calcification in moderate to late preterm birth category $(55.3 \%$ and $25.0 \%$ vs $29.4 \%$ and $17.6 \%$, respectively; $\mathrm{P}=0.012$ ). Chorionic villous necrosis prevailed in moderate to late preterm birth cases compared with extremely preterm, very preterm and term birth cases $(17.6 \%$ vs. $5.9 \%$ vs. $26.3 \%$ vs. $10.0 \%$; $\mathrm{P}=0.028$ ).

Strong association between gestational age and prematurely born neonatal morbidity was found (Figure 5). IVH, RDS, HIE and sepsis prevailed in extremely preterm and very preterm birth categories. As compared to preterm neonates without IVH, placental histological examination of preterm neonates with IVH more frequently revealed funisitis $(7.4 \%$ vs. $18.9 \%$, respectively; $\mathrm{P}=0.063)$. IUGR was linked to chorionic villous edema $(31.3 \%$ vs. $70.0 \%$, respectively; $\mathrm{P}=0.007)$ and villous necrosis $(50.2 \%$ vs. $92.0 \%$, respectively; $\mathrm{P}=0.014)$. Chorion-decidual hemorrhage and deciduitis was significantly associated with RDS ( $8.6 \%$ vs. $29.5 \%$, respectively; $\mathrm{P}=0.036$ and $7.1 \%$ vs. $28.4 \% ; \mathrm{P}=0.022$, respectively). The same association between RDS and chorionic villous fibrosis $(18.3 \%$ vs. $73.2 \%$, respectively; $\mathrm{P}=0.023$ ), chorionic villous hyalinization (14.2\% vs. $68.5 \%$, respectively; $\mathrm{P}=0.055)$, and chorionic villous calcification $(\mathrm{P}=0.084)$ was observed. Chorion-decidual hemorrhage was the main predisposing factor for HIE ( $43.4 \%$ vs. $59.6 \%$, respectively; $\mathrm{P}=0.058$ ). There was an association between $\mathrm{HIE}$ and chorionic villous hyalinization, fibrosis and calcification $(\mathrm{P}=0.022$; $\mathrm{P}=0.024$ and $\mathrm{P}=0.037$, respectively). Intervillous hemorrhage and decidual edema was verified as histologic counterpart of the fetal sepsis $(\mathrm{P}=0.017$ and $\mathrm{P}=0.061$, respectively).

\section{DISCUSSION}

The causes of singleton preterm births are incompletely understood. 5,6 Supporting the fetus through the progressive pregnancy, the placenta is a unique and valuable organ in explaining the pathogenesis of preterm birth. $^{5,6}$ Placental histological and microbiological assessment reveals different pathological findings in association with gestational age and neonatal morbidity. ${ }^{5,6}$

There is a large body of evidence that a cascade of activations of cellular components and mediators of inflammatory pathways results in onset of extremely preterm and very preterm births. ${ }^{13-15}$ The current study confirms that extremely preterm and very preterm births are associated with a high incidence of chorioamnionitis, deciduitis and funisitis that represents infection-related placental and umbilical cord lesions. Most cases of histopathological chorioamnionitis represent subclinical infection. Bacterial chorioamnionitis prevails in placentas in extremely preterm and very preterm birth categories, though does not influence the occurrence of neonatal morbidity controlling for gestational age. However, up to $20 \%$ of placentas in term birth have evidence of bacterial invasion and even inflammation. Based on the observation that $5-10 \%$ of placentas in term births have histological chorioamnionitis, some investigators suggest that chorioamnionitis may develop as consequence of preterm birth rather than representing a cause of preterm birth. ${ }^{5,13}$

Deciduitis is suggested to represent maternal response to chronic intrauterine colonisation or infection by organisms of low pathogenicity and predispose to preterm birth. ${ }^{13}$ Potentially, infection may develop early in gestation, before membrane fusion of the chorioamnion of the gestational sac to the opposite uterine wall at $19-20$ weeks, and then later after fusion, transmitted to the conceptus. ${ }^{13-15}$ In the literature, it is found that $40 \%$ of idiopathic preterm births placentas have chronic deciduitis. ${ }^{13-15}$ Further clinical and pathological studies may improve the understanding of the implications of this entity. ${ }^{13-15}$ 
The pathogenesis of preterm birth in the absence of infection may also involve activation of pathways responsible for normal onset of birth via components of the maternal and fetal hypothalamic-pituitary-adrenal axis. ${ }^{13,14}$ These include loss of the normally coordinated interactions and changes in systemic and local uterine balances of oxytocin levels, fetal cortisol levels, and decreasing estrogen to progesterone ratios, excessive uterine stretching, immunologically mediated processes and uterine anomalies. ${ }^{15-17}$ Other non-infectious triggers of preterm births are uteroplacental ischemia and oxidative stress. ${ }^{13-17}$

Placental abruption is defined as the sudden separation of a significant portion of placenta from its underlying maternal blood supply prior to delivery and is important cause of acute hypoxic injury. ${ }^{13,17,19}$ It is associated with a number of adverse outcomes including preterm birth, IUGR, HIE. ${ }^{17-20}$ It is often stated that the correlation between pathological and clinical abruption is poor. ${ }^{16-18}$ The gold standard for diagnosis of placental abruption is macroscopic appearance of retroplacental hemorrhage at the time of birth. ${ }^{5,15}$ The best pathological evidence is the gross finding of a retroplacental hematoma with either placental indentation or intraplacental extension. ${ }^{16,18}$ In the absence of these findings, microscopic evidence of interstitial hemorrhage in the basal plate or diffuse retromembranous hemorrhage is helpful to think about abruption. ${ }^{18,19}$ Finally, lesions associated with chronic maternal underperfusion are very commonly associated with abruption and can help strengthen a strong clinical suspicion of the diagnosis. ${ }^{13-17}$

As compared to the lower gestational age category, placentas in moderate to late preterm birth category predominantly show findings as hemorrhage and both umbilical cord vessels and placental plate thrombi, and degenerative changes in chorionic villi, reflecting disturbances of fetal-placental blood flow. It is currently believed that the major process leading to underperfusion is failure of trophoblast to appropriately invade and remodel the uterine spiral arteries. ${ }^{5,15}$ While the exact mechanisms of events leading to this outcome have not yet been explained, but a number of contributing factors have been identified. 5,15,19 Thrombo-occlusive lesions of large fetal vessels in the placenta and umbilical cord occur in the context of one or more of the classic triad of risk factors: vascular stasis, loss of surface resistance to coagulation, and circulatory hypercoagulability. ${ }^{15-17}$ Possible causes of fetal vascular stasis include prolonged umbilical cord obstruction, increased central venous pressure, and elevated level of hematocrit. ${ }^{13,17}$ It is likely that most cases of fetal thrombo-occlusive disease involve more than one risk factor. ${ }^{13,17}$

Sustained proximal vascular occlusion leads to degenerative changes in the distal chorionic villous tree. ${ }^{15-18}$ Longstanding occlusion of large arteries leads to distal chorionic hyalinized avascular villi. ${ }^{15-18}$ With longstanding venous obstruction, chorionic villi become hyalinized and avascular as with arterial obstruction. ${ }^{15,16}$ These chorionic villous changes can affect large or small groups of chorionic villi and can be localized or widely distributed throughout the placental parenchyma. ${ }^{15,19}$ Other lesions associated with fetal thrombo-occlusive disease include intimal fibrin cushions and fibromuscular sclerosis of arteries. ${ }^{15,19}$ Fibromuscular sclerosis represents concentric narrowing of the vascular lumen by proliferating smooth muscle cells and subendothelial fibroblasts, typically occurring in placental vessels lying between the point of occlusion and the affected chorionic villi secondary to lack of flow. ${ }^{5,15,19}$ Avascular chorionic villi are also associated with IUGR. ${ }^{15,19}$

All placental pathological findings have implications for the neonate and the mother. ${ }^{14-17}$ Placental infection has clinical significance for the neonate beyond just causing preterm birth. The fetus may reveal an inflammatory response associated with cytokine release that can cause damage to the developing brain and lungs. ${ }^{15,16}$ Inflammation of placenta and umbilical cord strongly correlates with the presence of high fetal levels of circulating pro-inflammatory cytokines and inflammatory mediators, such as interleukin- $6 .{ }^{15,16}$ This condition is referred to as the fetal inflammatory response syndrome. ${ }^{15,16}$ It is currently believed that various aspects of this response including circulating cytokines, bacterial toxins, and activation of the coagulation cascade predispose to adverse neurological outcomes, such as periventricular leukomalacia and cerebral palsy. ${ }^{17,20} \mathrm{~A}$ role for fetal inflammatory response syndrome in the development of chronic lung disease has also been found..$^{14,20,21}$ In the current study it is proposed that funisitis may help to predict the development of IVH, while placental findings as chorion-decidual hemorrhage and deciduitis may help to predict manifestation of RDS. A higher risk of developing IUGR was observed in preterm infants with intrauterine exposure to chorionic villous edema and villous fibrinoid necrosis. Therefore, chorionic villous edema and villous fibrinoid necrosis were identified as independent predictors for occurrence of IVH. In addition, in the literature chorionic villous edema is evidenced for cerebral palsy and impaired neurological function when these children reach school age. ${ }^{14,20,21}$ In the current study, it was concluded that chorion-decidual hemorrhage and chorionic villous hyalinization, fibrosis and calcification are strongly associated with the development of HIE. Thus, choriondecidual hemorrhage and chorionic villous hyalinization, fibrosis and calcification may help to predict the development of brain pathology among prematurely born neonates. Intervillous hemorrhage and decidual edema were verified as histological counterpart of neonatal sepsis.

Prenatal placental pathological assessment can be beneficial by searching for placental calcification, inflammatory lesions, thrombosis, hence should be evaluated prenatally by imaging studies or laboratory modalities due to the fact that they may lead to many 
instances of maternal and neonatal complications. ${ }^{13-16,19}$ It is suggested that pregnant women should be screened for placental pathological findings, and in case of any of these disorders appropriate treatment should be initiated to reduce placental pathology for the final goal of preventing preterm birth. ${ }^{5,18,19}$

Thus, placenta is not only a record of adverse conditions during intrauterine life that led preterm birth, it also likely holds clues to predicting which individual is at increased risk for developing short-term or long-term complication. ${ }^{5,14}$ In the case a specialist has suspicious about intrauterine inflammatory, thrombo-occlusive or hemorrhagic process, placenta in preterm birth should be examined for gross finding and histological findings urgently. Risk of respiratory or neurological complications in prematurely born neonate has been linked to specific histopathological pathology in placentas, hence histological and microbiological examination of placenta in preterm birth may provide important insights into risk assessment and future investigations. $^{14-16}$

According the study results, histological and microbiological examination of placenta in preterm birth, whenever possible, should be approached. The careful examination of placenta of prematurely born neonate, particularly in extremely preterm, should be part of routine obstetrical management to determine the causes of prematurity.

In conclusion, comprehension of the pathogenic mechanisms of prematurity of placenta and preterm birth, and the impact of placental prematurity on neonatal morbidity may lead to improved prenatal diagnostic and enhanced preventive care for both the mother and the child.

\section{Funding: No funding sources}

Conflict of interest: None declared

Ethical approval: The study was approved by the Institutional Ethics Committee

\section{REFERENCES}

1. World Health Organization Fact Sheet $N^{\circ} 363$. Available http://www.who.int/mediacentre/factsheets/fs363/en/ Accessed 13 October 2016.

2. The Centre for Disease Prevention and Control. Statistical Yearbook of Health Care in Latvia 16th edition, 2015. Available at: http://www.spkc.gov.lv/veselibas-aprupes-statistika/ Accessed 13 October 2016.

3. Azizi M, Rejaei M, Pezeshkpour Z, Alipour MR, Kazemi E, Zare Z wt al. Correlation Between Pathology of Placenta and Preterm Labor: A CaseControl Study J Biol Today's World. 2014;3(11):238-41.
4. Morgan TK. Role of the Placenta in Preterm Birth: A Review. Fetal Pediatr Pathol. 2016;9:1-8.

5. Ozer E. Placenta in Preterm Birth. Available at: http://www.intechopen.com/books/pretermbirth/placenta-in-preterm-birth Accessed 1 June 2016.

6. Chisholm KM, Heerema-McKenney A, Tian L, Rajani AK, Saria S, Koller D et al. Correlation of preterm infant illness severity with placental histology. Placenta. 2016;39:61-9.

7. Galinsky R, Polglase GR, Hooper SB, Black MJ, Moss TJM. The Consequences of Chorioamnionitis: Preterm Birth and Effects on Development. J of Pregn. 2013;1:1-11.

8. Goldenberg RL, Culhane JF, Iams JD, Romero R. Epidemiology and causes of preterm birth. Lancet. 2008:5;371(9606):75-84.

9. Royal College of Obstetricians and Gynaecologists. Fetal and perinatal pathology. Report of a joint working party. London, UK: RCOG-press; 2001.

10. Langston C, Kaplan C, Macpherson T, Manci E, Peevy K, Clark B, et al. Practice guideline for examination of the placenta: developed by the Placental Pathology Practice Guideline Development Task Force of the College of American Pathologists. Arch Pathol Lab Med. 1997;121:449-76.

11. Kim CJ, Romero R, Kusanovic JP, Yoo W, Dong Z, Topping V, et al. The Frequency, Clinical Significance, and Pathological Features of Chronic Chorioamnionitis: A Lesion Associated with Spontaneous Preterm Birth. Mod Pathol. 2010;23(7):1000-11.

12. Kim CJ, Romero R, Chaemsaithong P, Chaiyasit N, Yoon BH, Kim YM. Acute Chorioamnionitis and Funisitis: Definition, Pathologic Features, and Clinical Significance. Am J Obstet Gynecol. 2015;213(40):S29-52.

13. Fritsch MK. Placental pathology. Available at: https://pathology.uchicago.edu/sites/pathology.uchic ago.edu/files/uploads/PDFs/Placental\%20Pathology $\% 20$ Notes\%20Aspen\%202014\%20-

Fritsch\%20final.pdf Accessed 13 October 2016.

14. Stout MJ, Conlon B, Landeau M, Lee I, Bower C, Zhao $\mathrm{Q}$ et al. Identification of intracellular bacteria in the basal plate of the human placenta in term and preterm gestations. Am J Obstet Gynecol. 2013;208(3):226-6.

15. Dogan K, Salihoglu O, Sever N, Tombul T, Sari E, Yaşar L. Do Placental Histopathologic Characteristics Differ with Gestational Ages in Preterm and Term Deliveries? Fetal Pediatr Pathol. 2015;34(6):365-74.

16. Stanek J. Comparison of placental pathology in preterm, late-preterm, near-term, and term births. Am J Obstet Gynecol. 2014;210(3):234-44.

17. Mehta R, Nanjundaswamy S, Shen-Schwarz S, Petrova A. Neonatal Morbidity and Placental Pathology. Indian J Pediatr. 2006;73:25-8.

18. Beta J, Akolekar R, Ventura1 W, Syngelaki A, Nicolaides KH. Prediction of spontaneous preterm 
delivery from maternal factors, obstetric history and placental perfusion and function at 11-13 weeks. Prenat Diagn. 2011;31:75-83.

19. Stout MJ, Busam R, Macones GA, Tuuli MG. Spontaneous and Indicated Preterm Birth Subtypes: Inter-observer Agreement and Accuracy of Classification. Am J Obstet Gynecol. 2014;211(5):530-7.

20. Salafia CM, Charles AK, Maas EM. Placenta and fetal growth restriction. Clinics in Obstetrics and Gynecology. 2006;49:236-56.
21. Edmondson N, Bocking A, Machin G. The prevalence of chronic deciduitis in cases of preterm labour without clinical chorioamnionitis. Pediatric and Developmental Pathology. 2009;12:16-21.

Cite this article as: Kornete $\mathrm{A}$, Vedmedovska N, Blazuka S. Correlation between placental pathology and neonatal morbidity: a case-control study. Int J Reprod Contracept Obstet Gynecol 2017;6:599-605. 